Review

\title{
Determinants of voltage-gated sodium channel clustering in neurons
}

\author{
Christophe Leterrier ${ }^{\mathrm{a}, \mathrm{b}}$, Anna Brachet ${ }^{\mathrm{a}, \mathrm{b}}$, Bénédicte Dargent ${ }^{\mathrm{a}, \mathrm{b}}$, Helene Vacher ${ }^{\mathrm{a}, \mathrm{b}, *}$ \\ a INSERM UMR 641, Marseille F-13916, France \\ b Université de la Méditerranée, Faculté de Médecine Secteur-Nord, Institut Fédératif de Recherche 11, Marseille F-13916, France
}

\section{A R T I C L E I N F O}

Article history:

Available online $\mathrm{xxx}$

Keywords:

Axon initial segment

Node de Ranvier

Ankyrin

CK2

Endocytosis

\begin{abstract}
A B S T R A C T
In mammalian neurons, the generation and propagation of the action potential result from the presence of dense clusters of voltage-gated sodium channels (Nav) at the axonal initial segment (AIS) and nodes of Ranvier. In these two structures, the assembly of specific supra-molecular complexes composed of numerous partners, such as cytoskeletal scaffold proteins and signaling proteins ensures the high concentration of Nav channels. Understanding how neurons regulate the expression and discrete localization of Nav channels is critical to understanding the diversity of normal neuronal function as well as neuronal dysfunction caused by defects in these processes. Here, we review the mechanisms establishing the clustering of Nav channels at the AIS and in the node and discuss how the alterations of Nav channel clustering can lead to certain pathophysiologies.
\end{abstract}

(c) 2010 Elsevier Ltd. All rights reserved.

\section{Contents}

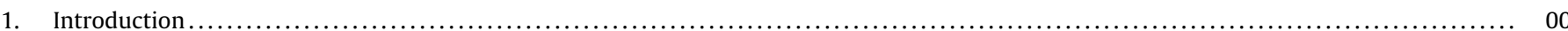

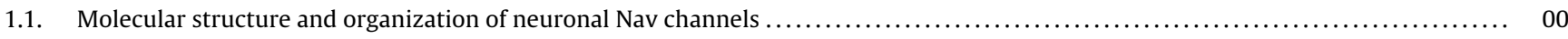

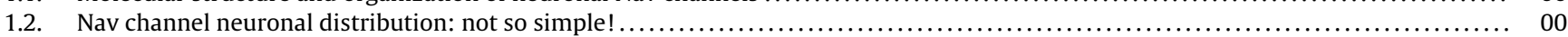

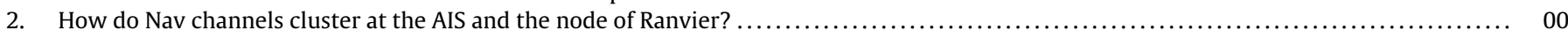

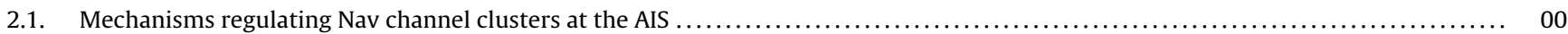

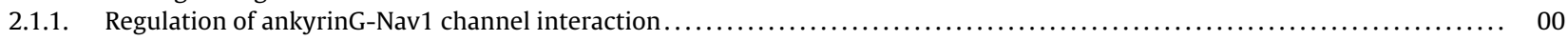

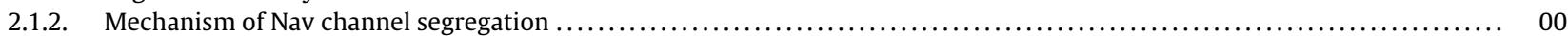

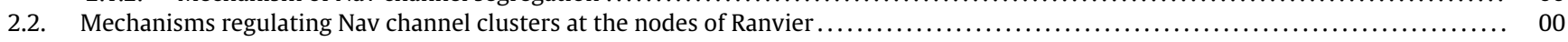

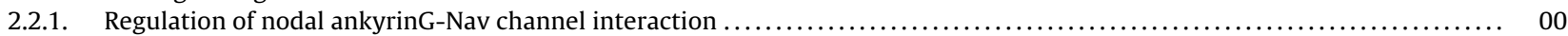

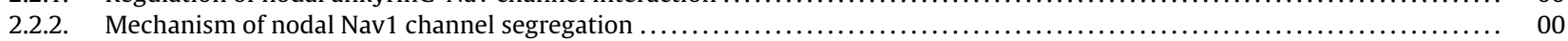

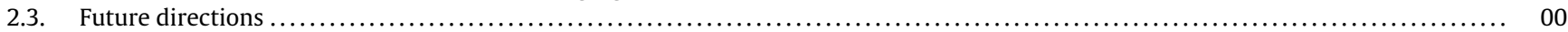

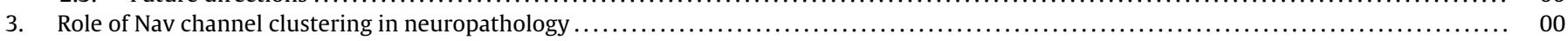

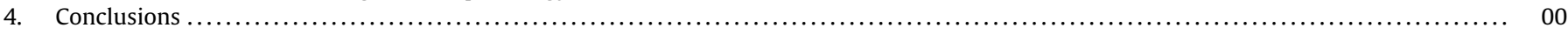

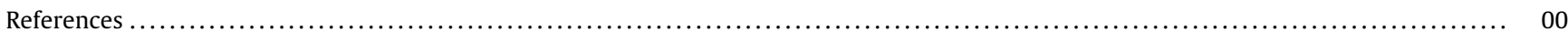

\section{Introduction}

The intrinsic electrical properties and the synaptic input-output relationships of neurons are governed by the action of a wide repertoire of ion channels. The localization of specific populations of ion channels with distinct functional properties at discrete sites in neurons dramatically impacts excitability and synaptic transmission. In mammalian neurons, dense clusters of voltage-gated

* Corresponding author at: Faculté de Médecine - Secteur Nord, Université de la Méditerranée CS80011, Bd Pierre Dramard 13344 Marseille Cedex 15, France. Tel.: +33 4916989 30; fax: +33491090506.

E-mail address: helene.vacher@univmed.fr (H. Vacher). sodium channels (Nav) at the axonal initial segment (AIS) and nodes of Ranvier underlie action potential generation and fast propagation [1,2] (Fig. 1). Nav clustering depends on the formation of supra-molecular complexes linked to the actin/spectrin cytoskeleton via the scaffold protein ankyrin G (ankG). Recently, much effort has focused on determining the molecular identity of these native neuronal Nav channel complexes, their cellular and subcellular distributions, as well as the mechanisms determining their discrete axonal subcellular distributions, as a crucial step in understanding their contribution to specific aspects of neuronal function. Here, we review progress made by recent studies aimed at determining the basic mechanisms of the specific targeting of Nav channels to the AIS and the node of Ranvier. We also discuss the currently asked questions and the implications for neuronal physiology. 


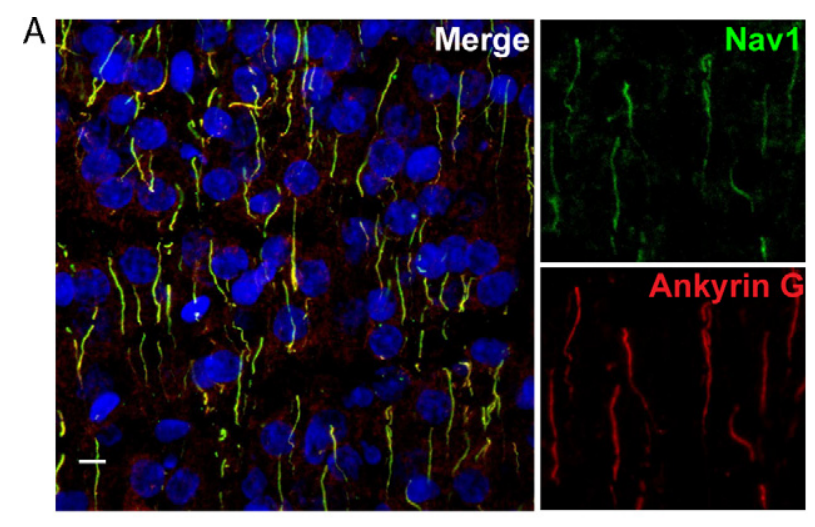

$\mathrm{B}$
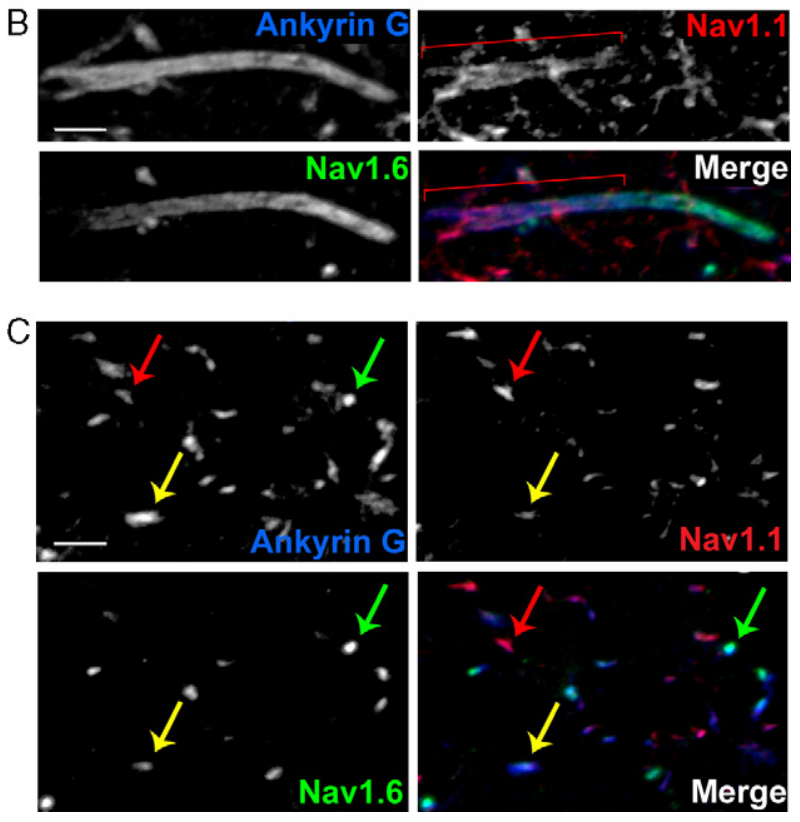

Fig. 1. In vivo localization of Nav channels. (A) In vivo concentration of Nav channels at the AIS of cortical neurons (green). Nav colocalizes at the AIS with ankG (red). Nuclei are labeled using Hoechst 33258 (blue). Scale bar, $10 \mu \mathrm{m}$. (B) Triple immunostaining of ankG, Nav1.1 and Nav1.6 in the adult lumbar spinal cord grey matter. Nav1.1 expression displayed a proximo-distal gradient, with a higher expression level in the AIS region proximal to the soma. Scale bar, $5 \mu \mathrm{m}$. (C) In vivo concentration of Nav1.1 and Nav1.6 in the nodes of Ranvier of the adult spinal cord white matter. Nav1.1 and Nav1.6 colocalized with ankG at the nodes. Three populations of nodes were identified: expressing Nav1.1 only (red arrows), Nav1.6 only (green arrows) or both (yellow arrows). Scale bar, $5 \mu \mathrm{m}$.

(B) and (C) panels were reproduced with permission from Molecular and Cellular Neuroscience; doi:10.1016/j.mcn.2008.06.008.

\subsection{Molecular structure and organization of neuronal Nav channels}

The first family of Nav channels contains all the classical voltage dependent sodium channels [3]. The outlying second family, termed Nax, contains channels whose sequences share some similarity to Nav1 sequences. In the mammalian genome, at least eight of the nine Nav1 $\alpha$ subunit genes (SCN1A (Nav1.1); SCN2A (Nav1.2); SCN3A (Nav1.3); SCN4A (Nav1.4); SCN5A (Nav1.5); SCN8A (Nav1.6); SCN9A (Nav1.7); SCN10A (Nav1.8); SCN11A (Nav1.9)) are expressed in the nervous system, the exception being the muscle-specific Nav1.4. Nav1.1, 1.2 and Nav1.6 are abundant in the central nervous system (CNS), while Nav1.3 is mostly present during embryonic development. Nav1.7, Nav1.8, and Nav1.9 are predominant in the peripheral nervous system (PNS) [12]. Nav channels consist of a highly processed $\alpha$ subunit, which is approximately $260 \mathrm{kDa}$, associated with auxiliary $\beta$ subunits $(\sim 30 \mathrm{kDa})$ through either covalent or non-covalent linkage [4]. The $\alpha$ subunits are organized into four homologous domains (I-IV), each of which contains six transmembrane $\alpha$ helices (S1-S6) and an additional pore loop located between the S5 and S6 segments. The pore loops line the outer, narrow entry to the pore, whereas the S5 and S6 segments line the inner, wider exit from the pore. The poreforming $\alpha$ subunit is sufficient for functional expression, but the kinetics and voltage dependence of channel gating are modulated by the $\beta$ subunits. These auxiliary subunits are involved in channel localization and interaction with cell adhesion molecules (CAMs), extracellular matrix (ECM), and intracellular cytoskeleton [5,6]. The role of $\beta$ subunits in neuronal Nav channel clustering will not be discussed in this review, for more details about their multiple roles see [7].

\subsection{Nav channel neuronal distribution: not so simple!}

Recent studies provide compelling evidences for a complex cell specific sub-domain organization of Nav1 subunits, with a spatially and temporally defined expression. In mature CNS and PNS nodes, Nav1.6 channels can be expressed either alone or with Nav1.1, while some nodes of Ranvier have been found expressing only Nav1.1 [8-10]. In some pathophysiological situations, Nav1.8, a subtype preferentially expressed in sensory neurons [3], can also be observed at nodes $[11,12]$. In addition, developmental changes occur at the nodes, where Nav1 subunits can all coexist at certain stages of maturation (e.g. nodes of the spinal cord [8]) (Fig. 1C). In mature retinal ganglion cell (RGC) axons, which consist of distinct unmyelinated and myelinated zones, Nav1.2 is found uniformly throughout unmyelinated regions, whereas Nav1.6 is specifically targeted to the nodes [13]. During the development of the RGCs, Nav1.2 is expressed first and clustered at the immature nodes of Ranvier, but as the myelination proceeds, Nav1.6 progressively replaces Nav1.2 at nodes [13].

In the same line, a cell-specific subcellular distribution of Nav1 subunits occurs at the AIS (Fig. 1). Nav1.6 can be exclusively and uniformly distributed along the AIS of Purkinje cells [14,15], or co-expressed with Nav1.1 at the AIS of cortical and cerebellar interneurons, in RGCs and in spinal cord neurons [8,14,16,17]. Moreover in these latter cells, Nav1.1 and Nav1.6 showed a complementary localization along the AIS: Nav1.1 subunits are found highly clustered at the proximal edge of the AIS, while Nav1.6 subunits exhibit an increase in density towards the proximal end of the AIS (Fig. 1B) $[8,18,19]$. In contrast, Nav1.1 and Nav1.6 channels are uniformly co-distributed along the AIS of main olfactory bulb short axon cells [14]. With regards to Nav1.2, it is co-expressed with Nav1.6 in layer 5 pyramidal cells of the neocortex with a complementary gradient of Nav1.2-Nav1.6 concentration along the AIS [20]. Nav1.2 and Nav1.6 are also co-expressed along the AIS of cerebellar granular cells (GCs) [21]. During development, Nav1.2 accumulation at the AIS precedes Nav1.6 in RGCs and in GCs [19,21]. It is important to note that these complex organizations of Nav1 channels at the AIS have a tremendous impact on the electrical properties of the cell. For example, in cortical pyramidal neurons, Nav1.6 accumulation at the distal AIS, determines the lowest threshold for AP initiation [20]. In contrast, a higher concentration of Nav1.2 channels in the proximal AIS promotes AP back propagation into the somadendritic compartment [20]. To complicate matters further, a recent study revealed, using a highly sensitive electron microscopic immunogold technique, the presence of the Nav1.6 subunit in the proximal and distal dendrites of hippocampal CA1 pyramidal cells. In these cells, Nav1.6 subunits have a lower density than that found in the AIS (by a factor of 35-80), and exhibit a gradual decrease in density along the proximodistal axis of the dendritic tree without being expressed in dendritic spines [22]. Consequently, 
further studies are required for a better understanding of the mechanisms accounting for such complex expressions and of the respective physiological relevance of each expression pattern.

\section{How do Nav channels cluster at the AIS and the node of Ranvier?}

The AIS and the node of Ranvier have a common molecular organization, due to multiple protein-rotein interactions where Nav channels are a core component of this interaction network [23]. Both sub-domains contain mainly ion channels (Nav, Kv7, and Kv1 only at the AIS) [14,24-26], CAMs (Neurofascin 186 (NF-186) and neuronal cell adhesion molecule (NrCAM)) [27-30], cytoskeletal adaptator complex like ankG and $\beta I V-s p e c t r i n ~[31-33]$, and ECM (brevican and versican). More recently, new proteins present at the AIS and the nodes have been identified: protein kinase CK2 [34], SCHIP-1 isoforms [35], FHF2 and FHF4 (two members of the fibroblast growth factor homologous factors (FHFs)) [36] and members of the NFKB signaling pathway [37,38]. Despite the molecular similarities between these two neuronal domains, there is one major difference between the AIS and the node of Ranvier: clustering of proteins at nodes requires the influence of myelinating glia (i.e. extrinsic factors), but recruitment of these same proteins to the AIS does not (i.e. intrinsic factors) [23]

\subsection{Mechanisms regulating Nav channel clusters at the AIS}

The AIS is intrinsically specified, being able to assemble in cultured neurons in the absence of glial cells [39-41]. In vivo and in vitro analysis all demonstrate a crucial role of ankG as the master organizer for AIS assembly and maintenance [30,33,42]. AnkG is the first component localized at the AIS [42], and through its membrane-binding domain (MBD) (ankyrin repeat domain), specifically clusters Nav [43,44], Kv7.2/7.3 channels [25] and CAMs [45], as well as the scaffolding protein $\beta I V$-spectrin through its spectrin domain [46]. In fact, mice lacking ankG in Purkinje cells fail to cluster any other AIS components [33,42], and electroporation of cortical neurons with shRNA against ankG blocks AIS assembly [30]. In cultured hippocampal neurons, knockdown of ankG impedes AIS formation, while knockdowns of CAMs, Nav channels or BIV-spectrin have little impact [30]. A role for Nav channels in AIS formation and maintenance is, however, not excluded. In cultured motor neurons, the decrease of Nav channel expression affects AIS assembly, suggesting that a complementary mechanism might exist in this cell type [47]. However, the in vivo confirmation of the role of Nav channel in AIS assembly may prove difficult, because of the perinatal lethality of the Nav1.2 knockout mouse [48] and the compensation of Nav1.6 disappearance by Nav1.2 and Nav1.1 in the medJ mouse [15].

\subsubsection{Regulation of ankyrinG-Nav1 channel interaction}

AnkG has been known to link Nav channels to the underlying AIS cytoskeleton, but the molecular details of this interaction and its role in Nav channel concentration at the AIS were only recently unraveled $[34,43,44,49,50]$. The use of chimeric protein expression in cultured hippocampal neurons showed that the cytoplasmic loop linking domains II and III was responsible for the AIS concentration of Nav channels [43]. This II-III loop was sufficient to either segregate a chimera of the CD4 protein (CD4-Nav1.2) at the AIS, or redirect the somatodendritic potassium channel Kv2.1 (Kv2.1-Nav1.2) to the AIS. Two independent studies revealed that the AIS targeting was abolished upon truncation of a sequence of 27 residues within the Nav1.2 II-III loop (a.a. 1102-1128) [43], and that the PIALGESD sequence located within these 27 residues directly interacted with the MBD of ankG [44]. However, this 27residue sequence, called ank-binding domain, is not only specific to ankG, but it also allows binding to ankyrin-B (ankB), which shares the canonical MBD. AnkB is located along the distal axon in cultured hippocampal neurons and in myelinated fibers [31]. Thus, this suggests the existence of an additional mechanism ensuring the specific interaction of Nav channels with ankG at the AIS. Recent findings showed that specific residues within the ankbinding domain are phosphorylated by the protein kinase CK2, allowing for a high-affinity recognition of ankG [34]. In vitro assays demonstrated that the CK2-mediated phosphorylation of the ankbinding domain of neuronal Nav channels resulted in a 1000-fold increase in binding affinity for the MBD of ankG (from micromolar to nanomolar). Although, in this study CK2-mediated phosphorylation equally enhanced the affinities for either the MBD of ankG or ankB, the authors showed that CK2 was highly concentrated at the AIS (Fig. 2A and B) and that inhibition of CK2 activity disrupted the AIS localization of Nav channels [34]. This implies that the local concentration of CK2 enhances the interaction of Nav channels with AnkG via their phosphorylations, contributing to their specific accumulation at the AIS. However, the mechanisms regulating the AIS targeting and timing of CK2 as well as the phosphatase counterbalancing of CK2 phosphorylation are still unknown. AnkG also modulates the inactivation properties of $\mathrm{Na}+$ current in heterologous cells [51]. However, the electrophysiological consequences of a phosphodependant binding of ankG remain to be explored in neurons. Finally, it is important to note that the ank-binding domain is highly conserved among Nav channels and that some neuron-specific subtypes of Nav channel can be present in the somatodendritic domain. Therefore, it is likely that some Nav channels bear an additional determinant that overrides the Ank-binding domain.

\subsubsection{Mechanism of Nav channel segregation}

Some important questions concerning the targeting of Nav channels to the AIS still remain: are the Nav channels directly inserted into the AIS plasma membrane from biosynthetic vesicles? Or, are the channels initially transported and inserted in the whole neuronal membrane, before being subsequently retrieved from the dendrites, soma and the distal axon by endocytosis? Are these two models mutually exclusive or co-existing at some stage of the neuronal development? Studies have shown that in developing neurons, endogenous Nav channels can undergo activity-dependent endocytosis [52,53]. In fact, Nav subunits possess two endocytosis signals located in the C-terminal tail [54] and in the proximal region of the II-III loop [50]. CD4-chimeric proteins bearing the C-terminal tail of Nav channels are localized to the axonal plasma membrane in dissociated hippocampal neurons. This compartmentalization arises from the selective endocytosis of the chimera in the somatodendritic compartment [54]. In a similar fashion, the same reporter bearing the Nav1.2 II-III loop is first inserted in the somatodendritic compartment and distal axon, before being endocytosed from these sites and restricted to the AIS [50]. One model arising from these findings is that in developing neurons, an elimination/retention mechanism accounts for Nav channel compartimentalization in axons. Nav channels are first sorted indiscriminately to the whole neuronal plasma membrane. These channels would then diffuse laterally until they are endocytosed or trapped by ankG via their ank-binding domain when they reach the AIS (Fig. 2C). It is also possible that the two endocytosis signals lead to differential regulations of endocytosis in the somatodenditric compartment versus the axon. In addition, the presence of a conserved binding site for Nedd 4 and Nedd4-2 ubiquitin ligases in the C-terminus of several Nav1 subunits suggests that endocytosed channels are targeted to degradative pathways [55]. 


\subsection{Mechanisms regulating Nav channel clusters at the nodes of Ranvier}

The mechanisms of node formation and maintenance are quite complex due to the presence of myelinating cells: Schwann cells for the PNS and oligodendrocytes for the CNS [56,57]. In the PNS, the nodes are first formed, followed by paranodes and juxtaparanodes [58-60]. In vitro studies showed that Schwann cells secrete gliomedin, an oligomeric ligand that recruits first the adhesion protein NF-186 to nascent nodes [57]. NF-186 is then able to recruit ankG to nodes, which in turn recruits Nav channels [29] and $\beta I V-$ spectrin [46]. This sequence of events, and the prominent role of NF-186 in PNS node assembly, is confirmed by the general absence of nodes observed in NF-186 knockout mice [61]. Moreover, mice deficient for nodal components not directly involved in node assembly, such as NrCAM [62] and BIV-spectrin [45,63,64], also exhibit altered nodes, demonstrating the interdependence of nodal component for long-term nodal maintenance.

The mechanisms of node formation in the CNS are not as understood as in the PNS, mainly because of the lack of in vitro models. As gliomedin is absent in the CNS, it has been proposed that a redundant network of ECM proteins play a role in the initiation of NF-186 recruitment [56], and that the clustering of Nav channels is induced in RGC cultures by an unidentified soluble factor released by oligodendrocytes [21]. In addition, in contrast to the PNS, the concentration of paranodal components occurs before nodal ones [65], and ankG clusters can appear before adhesion proteins at the optic nerve nodes [66]. This suggests the presence of complementary or alternative mechanisms directing the assembly of CNS nodes
([56], see below), where the requirement for NF-186 is not crucial [67].

\subsubsection{Regulation of nodal ankyrinG-Nav channel interaction}

All the mechanisms described above, place ankG and Nav channels as downstream participants in node formation. The effect of ankG absence at nodes in vivo is unknown, as the current cerebellar ankG knockout mouse model still exhibits ankG labeling at nodes of Ranvier [42]. In myelinated DRG cultures however, knockdown of ankG in neurons abolishes node formation, including NF-186 clustering, indicating a central role for ankG in maintaining the integrity of the nodal complex [29]. Although CK2 is concentrated at the nodes of Ranvier in the CNS and PNS [34], its role in directly regulating the ankG-Nav interaction has not yet been determined.

\subsubsection{Mechanism of nodal Nav1 channel segregation}

The trafficking mechanisms allowing for delivery and concentration of Nav channels in the nodes of Ranvier have yet to be unraveled. It is likely that nodal membrane proteins are directly inserted into the node where they are confined because of the presence of paranodes and their junctions [65,67-70]. Paranodal junctions limit subsequent diffusion of nodal membrane proteins, in particular, Nav channels [71]. Several mouse models with altered paranodal junctions have longer and more diluted clusters of Nav channels [68-70]. Strikingly, paranodal junctions also have a prominent role in the differential expression of Nav1.2 and Nav1.6 subunits at nodes $[13,68]$, and mice with altered paranodes exhibit incomplete replacement of Nav1.2 by Nav1.6 in PNS nodes $[68,70]$. The internode region, where the axonal membrane is apposed to
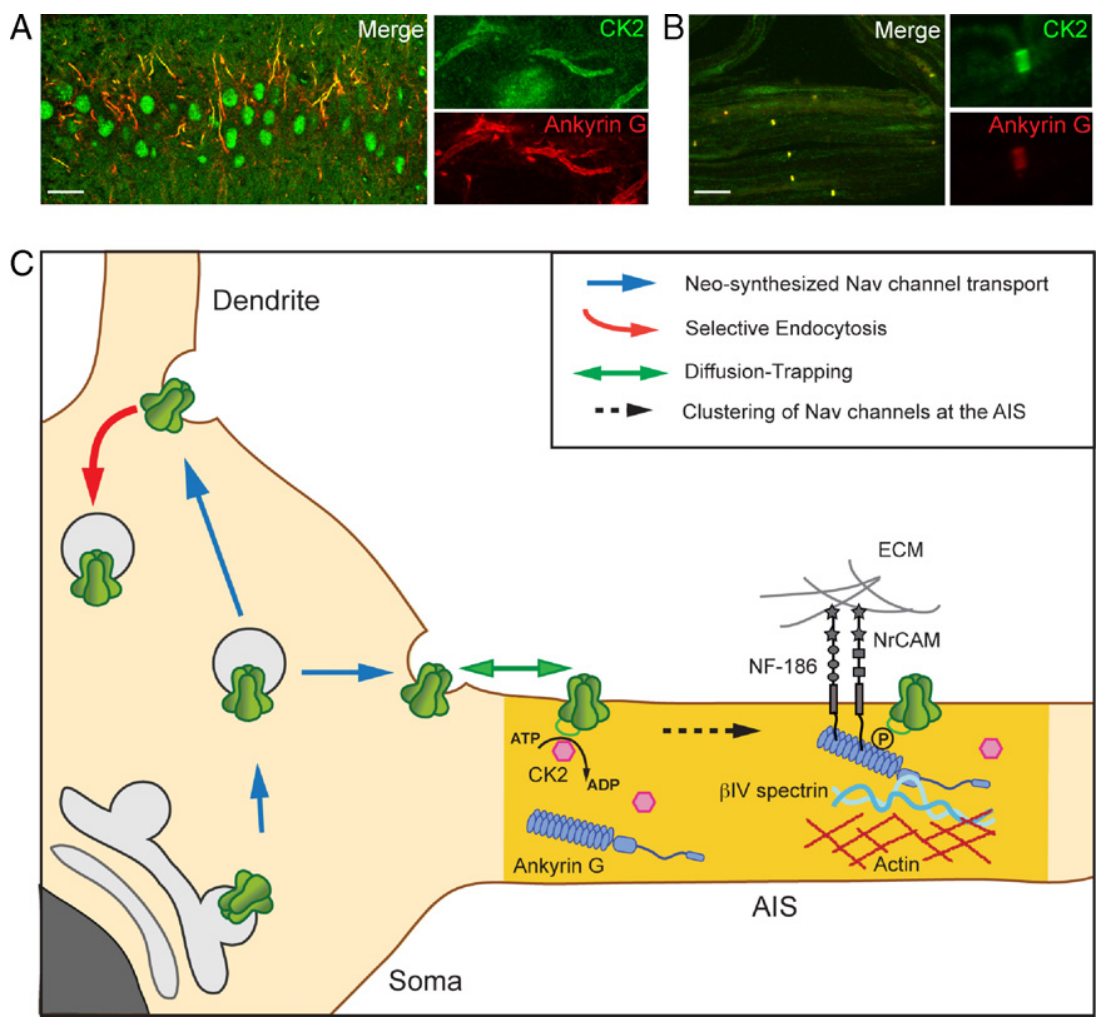

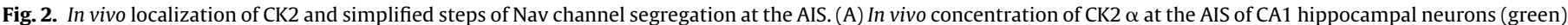

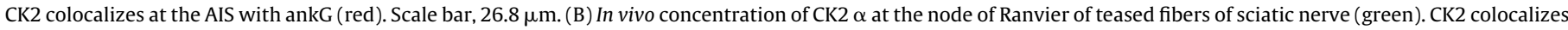

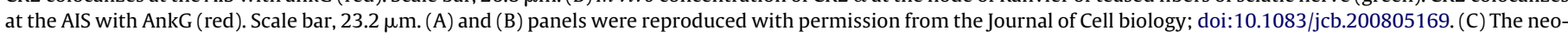

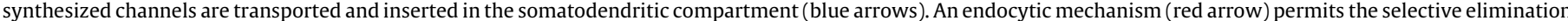

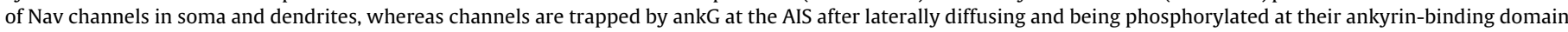

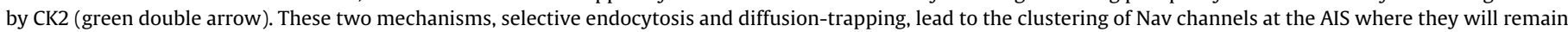
firmly anchored via the actin-spectrin-ankG based cytoskeleton and the adhesion molecules (NF-186 and NrCAM)-ECM network. 
glial cells, has recently been implicated in Nav channel clustering as it actively forces Nav channels toward the nodes [72]. Finally, the fact that Nav1.2, but not Nav1.6, can cluster to form immature nodes in RGC cultures treated with oligodendrocyte-conditioned medium [73] further suggests that myelinating cells could direct the differential targeting of the two Nav subunits at the nodes.

\subsection{Future directions}

The key ankyrin-binding and endocytosis motifs are conserved between neuronal Nav subunits (Nav1.1-3 and Nav1.6). However, these subunits are present in other compartments such as soma, dendrites and synapses with a lower density than that found in the AIS and the nodes [9,22]. In the same line, all the Nav1.1, Nav1.2 and Nav1.6 subunits bear the same set of CK2 phosphorylation sites in their ank-binding domain, but they are differentially expressed during development, and exhibit distinct cell type-dependent subdomain organizations along the AIS $[14,17,20]$. Therefore, this suggests the presence of additional mechanisms, which can overcome all the mechanisms described above in a cell type-dependent manner. Different motifs and/or binding-partners, not identified yet, could underlie these preferential sub-cellular and cell-type dependent organizations. Intercellular and intracellular signaling, transcription factors, and micro-RNAs could also control the developmental regulations.

Finally, recent studies have discovered that the AIS can be surprisingly plastic in its responses to ongoing levels and patterns of electrical activity $[74,75]$ as well as to alterations in neuronal health [76]. This plasticity results in different changes in terms of length, position and composition of the AIS. For instance, in avian auditory neurons, the length of the AIS, defined by the distribution of Nav channels and ankG, increases according to the levels of presynaptic activity [75]. However, the mechanisms underlying such modifications of local Nav channel densities and/or distributions are poorly understood. It is likely that CK2 could play a role in the activity-driven AIS plasticity.

\section{Role of Nav channel clustering in neuropathology}

It is important to understand the mechanisms directing Nav channel targeting to the AIS and nodes, as improper localization or expression of Nav channels has been implicated in a number of pathological conditions linked to altered conduction and electrogenic properties of neurons [77]. Strikingly, defects in Nav channel function can lead to either neurological hyperexcitability [78] or conduction failure/axonal degeneration [79] depending on the pathological context. Mutations in Nav1.1, and to a lesser extent Nav1.2, have been detected in several epileptic syndromes, namely Generalized Epilepsy with Febrile Seizures Plus (GEFS+), Sever Myoclonic Epilepsy of Infancy (SMEI) and Benign Familial Neonatal Infantile Seizures (BFNIS). Nav1.1 mutants seem to be mostly loss-of-function, primarily affecting inhibitory interneurons where Nav1.1 is highly expressed $[8,14]$. The resulting defect in interneuron excitability is likely to explain the global hyperexcitability and the seizure phenotype associated with these mutations in mouse models and epileptic patients [80]. In contrast, some Nav1.2 mutations have been associated with impaired trafficking of channels to the plasma membrane, but it is not clear how a Nav1.2 loss of function can lead to hyperexcitability.

Beyond the pathological consequences of mutations in Nav channels, perturbations of Nav expression and localization has been implicated in demyelinating diseases such as multiple sclerosis [81]. Nav channel concentration at nodes is lost during demyelination, and Nav channels diffuse all along demyelinating segments. Not only Nav1.6, but also Nav1.2 is often detected in these axonal segments, suggesting that new channels are expressed and inserted along the unmyelinated membrane. The consequence of this mislocalization can be beneficial, as this low concentration of Nav channels can restore conduction along demyelinated segments. However, it has been suggested that expression of Nav1.6, which exhibits a significant persistent current, plays a role in axonal degeneration: the resultant Nav loading in axons is responsible for activation of the $\mathrm{Na}+/ \mathrm{Ca}^{2+}$ exchanger and subsequent calcium-mediated axonal damage [82]. Moreover, a misexpression of non-CNS channels also occurs in neurons during MS, as shown for Purkinje cells expressing Nav1.8 in MS patients and mouse models of MS [83].

\section{Conclusions}

It is now clear that Nav channel densities and discrete localizations are dynamically regulated, as was illustrated by the recent characterization of the kinase CK2 [34]. However, to answer to all the questions posed above, it will be absolutely necessary to make a great effort in developing new model systems and to combine them with advanced electrophysiological, imaging and molecular methods. These new approaches will surely reveal the intricate set of mechanisms that allows for a fine-tuning of neuronal excitability and plasticity in normal and pathological conditions.

\section{References}

[1] Clark BD, Goldberg EM, Rudy B. Electrogenic tuning of the axon initial segment. Neuroscientist 2009;15:651-68.

[2] Kress GJ, Mennerick S. Action potential initiation and propagation: upstream influences on neurotransmission. Neuroscience 2009;158:211-22.

[3] Catterall WA, Goldin AL, Waxman SG. International union of pharmacology. XLVII. Nomenclature and structure-function relationships of voltage-gated sodium channels. Pharmacol Rev 2005;57:397-409.

[4] Catterall WA. From ionic currents to molecular mechanisms: the structure and function of voltage-gated sodium channels. Neuron 2000;26:13-25.

[5] Qu Y, Curtis R, Lawson D, Gilbride K, Ge P, DiStefano PS, et al. Differential modulation of sodium channel gating and persistent sodium currents by the beta1, beta2, and beta3 subunits. Mol Cell Neurosci 2001;18:570-80.

[6] McEwen DP, Meadows LS, Chen C, Thyagarajan V, Isom LL. Sodium channel beta1 subunit-mediated modulation of Nav1.2 currents and cell surface density is dependent on interactions with contactin and ankyrin. J Biol Chem 2004;279:16044-9.

[7] Patino GA, Isom LL. Electrophysiology and beyond: multiple roles of $\mathrm{Na}(+)$ channel beta subunits in development and disease. Neurosci Lett 2010. Jun 23 [Epub ahead of print].

[8] Duflocq A, Le Bras B, Bullier E, Couraud F, Davenne M. Nav1.1 is predominantly expressed in nodes of Ranvier and axon initial segments. Mol Cell Neurosci 2008;39:180-92.

[9] Vacher H, Mohapatra DP, Trimmer JS. Localization and targeting of voltagedependent ion channels in mammalian central neurons. Physiol Rev 2008;88:1407-47.

[10] Caldwell JH, Schaller KL, Lasher RS, Peles E, Levinson SR. Sodium channel $\mathrm{Na}(\mathrm{v}) 1.6$ is localized at nodes of ranvier, dendrites, and synapses. Proc Natl Acad Sci USA 2000;97:5616-20.

[11] Devaux JJ, Scherer SS. Altered ion channels in an animal model of Charcot-Marie-Tooth disease type IA. J Neurosci 2005;25:1470-80.

[12] Arroyo EJ, Xu T, Grinspan J, Lambert S, Levinson SR, Brophy PJ, et al. Genetic dysmyelination alters the molecular architecture of the nodal region. J Neurosci 2002;22:1726-37.

[13] Boiko T, Rasband MN, Levinson SR, Caldwell JH, Mandel G, Trimmer JS, et al. Compact myelin dictates the differential targeting of two sodium channel isoforms in the same axon. Neuron 2001;30:91-104.

[14] Lorincz A, Nusser Z. Cell-type-dependent molecular composition of the axon initial segment. J Neurosci 2008;28:14329-40.

[15] Van Wart A, Matthews G. Impaired firing and cell-specific compensation in neurons lacking nav1.6 sodium channels. J Neurosci 2006;26:7172-80.

[16] Ogiwara I, Miyamoto H, Morita N, Atapour N, Mazaki E, Inoue I, et al. Na(v)1.1 localizes to axons of parvalbumin-positive inhibitory interneurons: a circuit basis for epileptic seizures in mice carrying an Scn1a gene mutation. J Neurosci 2007;27:5903-14.

[17] Van Wart A, Trimmer JS, Matthews G. Polarized distribution of ion channels within microdomains of the axon initial segment. J Comp Neurol 2007;500:339-52.

[18] Van Wart A, Matthews G. Expression of sodium channels Nav1.2 and Nav1.6 during postnatal development of the retina. Neurosci Lett 2006;403:315-7.

[19] Boiko T, Van Wart A, Caldwell JH, Levinson SR, Trimmer JS, Matthews G. Functional specialization of the axon initial segment by isoform-specific sodium channel targeting. J Neurosci 2003;23:2306-13. 
[20] Hu W, Tian C, Li T, Yang M, Hou H, Shu Y. Distinct contributions of Na(v)1.6 and $\mathrm{Na}(\mathrm{v}) 1.2$ in action potential initiation and backpropagation. Nat Neurosci 2009;12:996-1002.

[21] Osorio N, Cathala L, Meisler MH, Crest M, Magistretti J, Delmas P. Persistent Nav1.6 current at axon initial segments tunes spike timing of cerebellar granule cells. J Physiol 2010;588:651-70.

[22] Lorincz A, Nusser Z. Molecular identity of dendritic voltage-gated sodium channels. Science 2010;328:906-9.

[23] Hedstrom KL, Rasband MN. Intrinsic and extrinsic determinants of ion channel localization in neurons. J Neurochem 2006;98:1345-52.

[24] Devaux JJ, Kleopa KA, Cooper EC, Scherer SS. KCNQ2 is a nodal K+ channel. J Neurosci 2004;24:1236-44.

[25] Pan Z, Kao T, Horvath Z, Lemos J, Sul JY, Cranstoun SD, et al. A common ankyrin-G-based mechanism retains KCNQ and NaV channels at electrically active domains of the axon. J Neurosci 2006;26:2599-613.

[26] Inda MC, DeFelipe J, Munoz A. Voltage-gated ion channels in the axon initial segment of human cortical pyramidal cells and their relationship with chandelier cells. Proc Natl Acad Sci USA 2006;103:2920-5.

[27] Basak S, Raju K, Babiarz J, Kane-Goldsmith N, Koticha D, Grumet M. Differential expression and functions of neuronal and glial neurofascin isoforms and splice variants during PNS development. Dev Biol 2007;311:408-22.

[28] Davis JQ Lambert S, Bennett V. Molecular composition of the node of Ranvier: identification of ankyrin-binding cell adhesion molecules neurofascin (mucin+/third FNIII domain-) and NrCAM at nodal axon segments. J Cell Biol 1996;135:1355-67.

[29] Dzhashiashvili Y, Zhang Y, Galinska J, Lam I, Grumet M, Salzer JL. Nodes of Ranvier and axon initial segments are ankyrin G-dependent domains that assemble by distinct mechanisms. J Cell Biol 2007;177:857-70.

[30] Hedstrom KL, Xu X, Ogawa Y, Frischknecht R, Seidenbecher CI, Shrager P, et al. Neurofascin assembles a specialized extracellular matrix at the axon initial segment. J Cell Biol 2007;178:875-86.

[31] Bennett V, Healy J. Membrane domains based on ankyrin and spectrin associated with cell-cell interactions. Cold Spring Harb Perspect Biol 2009; 1:a003012.

[32] Salzer JL. Polarized domains of myelinated axons. Neuron 2003;40:297-318.

[33] Zhou D, Lambert S, Malen PL, Carpenter S, Boland LM, Bennett V. AnkyrinG is required for clustering of voltage-gated Na channels at axon initial segments and for normal action potential firing. J Cell Biol 1998;143:1295-304.

[34] Brechet A, Fache MP, Brachet A, Ferracci G, Baude A, Irondelle M, et al. Protein kinase CK2 contributes to the organization of sodium channels in axonal membranes by regulating their interactions with ankyrin G. J Cell Biol 2008;183:1101-14.

[35] Martin PM, Carnaud M, Garcia del Cano G, Irondelle M, Irinopoulou T, Girault JA, et al. Schwannomin-interacting protein-1 isoform IQCJ-SCHIP-1 is a late component of nodes of Ranvier and axon initial segments. J Neurosci 2008;28:6111-7.

[36] Goldfarb M. Fibroblast growth factor homologous factors: evolution, structure, and function. Cytokine Growth Factor Rev 2005;16:215-20.

[37] Schultz C, Konig HG, Del Turco D, Politi C, Eckert GP, Ghebremedhin E, et al. Coincident enrichment of phosphorylated IkappaBalpha, activated IKK, and phosphorylated p65 in the axon initial segment of neurons. Mol Cell Neurosci 2006;33:68-80.

[38] Politi C, Del Turco D, Sie JM, Golinski PA, Tegeder I, Deller T, et al. Accumulation of phosphorylated I kappaB alpha and activated IKK in nodes of Ranvier. Neuropathol Appl Neurobiol 2008;34:357-65.

[39] Winckler B, Forscher P, Mellman I. A diffusion barrier maintains distribution of membrane proteins in polarized neurons. Nature 1999;397:698-701.

[40] Catterall WA. Localization of sodium channels in cultured neural cells. J Neurosci 1981;1:777-83.

[41] Zhang X, Bennett V. Restriction of $480 / 270-k D a$ ankyrin G to axon proximal segments requires multiple ankyrin G-specific domains. J Cell Biol 1998; 142:1571-81.

[42] Jenkins SM, Bennett V. Ankyrin-G coordinates assembly of the spectrin-based membrane skeleton, voltage-gated sodium channels, and L1 CAMs at Purkinje neuron initial segments. J Cell Biol 2001;155:739-46.

[43] Garrido JJ, Giraud P, Carlier E, Fernandes F, Moussif A, Fache MP, et al. A targeting motif involved in sodium channel clustering at the axonal initial segment. Science 2003;300:2091-4.

[44] Lemaillet G, Walker B, Lambert S. Identification of a conserved ankyrinbinding motif in the family of sodium channel alpha subunits. J Biol Chem 2003;278:27333-9.

[45] Yang Y, Lacas-Gervais S, Morest DK, Solimena M, Rasband MN. BetaIV spectrins are essential for membrane stability and the molecular organization of nodes of Ranvier. J Neurosci 2004;24:7230-40.

[46] Yang Y, Ogawa Y, Hedstrom KL, Rasband MN. betaIV spectrin is recruited to axon initial segments and nodes of Ranvier by ankyrinG. J Cell Biol 2007;176:509-19.

[47] Xu X, Shrager P. Dependence of axon initial segment formation on $\mathrm{Na}+$ channel expression. J Neurosci Res 2005;79:428-41.

[48] Planells-Cases R, Caprini M, Zhang J, Rockenstein EM, Rivera RR, Murre C, et al. Neuronal death and perinatal lethality in voltage-gated sodium channel alpha(II)-deficient mice. Biophys J 2000;78:2878-91.

[49] Hill AS, Nishino A, Nakajo K, Zhang G, Fineman JR, Selzer ME, et al. Ion channel clustering at the axon initial segment and node of Ranvier evolved sequentially in early chordates. PLoS Genet 2008;4:e1000317.

[50] Fache MP, Moussif A, Fernandes F, Giraud P, Garrido JJ, Dargent B. Endocytotic elimination and domain-selective tethering constitute a potential mechanism of protein segregation at the axonal initial segment. J Cell Biol 2004;166:571-8.

[51] Shirahata E, Iwasaki H, Takagi M, Lin C, Bennett V, Okamura Y, et al. Ankyrin-G regulates inactivation gating of the neuronal sodium channel, Nav1.6. J Neurophysiol 2006;96:1347-57.

[52] Dargent B, Paillart C, Carlier E, Alcaraz G, Martin-Eauclaire MF, Couraud F. Sodium channel internalization in developing neurons. Neuron 1994;13:683-90.

[53] Dargent B, Couraud F. Down-regulation of voltage-dependent sodium channels initiated by sodium influx in developing neurons. Proc Natl Acad Sci USA 1990;87:5907-11.

[54] Garrido JJ, Fernandes F, Giraud P, Mouret I, Pasqualini E, Fache MP, et al. Identification of an axonal determinant in the C-terminus of the sodium channel $\mathrm{Na}(\mathrm{v}) 1.2$. EMBO J 2001;20:5950-61.

[55] Fotia AB, Ekberg J, Adams DJ, Cook DI, Poronnik P, Kumar S. Regulation of neuronal voltage-gated sodium channels by the ubiquitin-protein ligases Nedd 4 and Nedd4-2. J Biol Chem 2004;279:28930-5.

[56] Susuki K, Rasband MN. Molecular mechanisms of node of Ranvier formation. Curr Opin Cell Biol 2008;20:616-23.

[57] Eshed Y, Feinberg K, Poliak S, Sabanay H, Sarig-Nadir O, Spiegel I, et al. Gliomedin mediates Schwann cell-axon interaction and the molecular assembly of the nodes of Ranvier. Neuron 2005;47:215-29.

[58] Melendez-Vasquez CV, Rios JC, Zanazzi G, Lambert S, Bretscher A, Salzer JL. Nodes of Ranvier form in association with ezrin-radixin-moesin(ERM)-positive Schwann cell processes. Proc Natl Acad Sci USA 2001;98:1235-40.

[59] Poliak S, Gollan L, Salomon D, Berglund EO, Ohara R, Ranscht B, et al. Localization of Caspr2 in myelinated nerves depends on axon-glia interactions and the generation of barriers along the axon. J Neurosci 2001;21:7568-75.

[60] Salzer JL, Brophy PJ, Peles E. Molecular domains of myelinated axons in the peripheral nervous system. Glia 2008;56:1532-40.

[61] Sherman DL, Tait S, Melrose S, Johnson R, Zonta B, Court FA, et al. Neurofascins are required to establish axonal domains for saltatory conduction. Neuron 2005;48:737-42.

[62] Custer AW, Kazarinova-Noyes K, Sakurai T, Xu X, Simon W, Grumet M, et al. The role of the ankyrin-binding protein NrCAM in node of Ranvier formation. J Neurosci 2003;23:10032-9.

[63] Lacas-Gervais S, Guo J, Strenzke N, Scarfone E, Kolpe M, Jahkel M, et al. BetaIVSigma1 spectrin stabilizes the nodes of Ranvier and axon initial segments. J Cell Biol 2004;166:983-90.

[64] Komada M, Soriano P. [Beta]IV-spectrin regulates sodium channel clustering through ankyrin-G at axon initial segments and nodes of Ranvier. J Cell Biol 2002;156:337-48.

[65] Rasband MN, Peles E, Trimmer JS, Levinson SR, Lux SE, Shrager P. Dependence of nodal sodium channel clustering on paranodal axoglial contact in the developing CNS. J Neurosci 1999;19:7516-28.

[66] Jenkins SM, Bennett V. Developing nodes of Ranvier are defined by ankyrin-C clustering and are independent of paranodal axoglial adhesion. Proc Natl Acad Sci USA 2002;99:2303-8

[67] Zonta B, Tait S, Melrose S, Anderson H, Harroch S, Higginson J, et al. Glial and neuronal isoforms of Neurofascin have distinct roles in the assembly of nodes of Ranvier in the central nervous system. J Cell Biol 2008;181:1169-77.

[68] Rios JC, Rubin M, St Martin M, Downey RT, Einheber S, Rosenbluth J, et al. Paranodal interactions regulate expression of sodium channel subtypes and provide a diffusion barrier for the node of Ranvier. J Neurosci 2003;23:7001-11.

[69] Rosenbluth J, Dupree JL, Popko B. Nodal sodium channel domain integrity depends on the conformation of the paranodal junction, not on the presence of transverse bands. Glia 2003;41:318-25

[70] Suzuki A, Hoshi T, Ishibashi T, Hayashi A, Yamaguchi Y, Baba H. Paranoda axoglial junction is required for the maintenance of the Nav1.6-type sodium channel in the node of Ranvier in the optic nerves but not in peripheral nerve fibers in the sulfatide-deficient mice. Glia 2004:46:274-83.

[71] Rosenbluth J. Intramembranous particle distribution at the node of Ranvier and adjacent axolemma in myelinated axons of the frog brain. J Neurocytol 1976;5:731-45.

[72] Voas MG, Glenn TD, Raphael AR, Talbot WS. Schwann cells inhibit ectopic clustering of axonal sodium channels. J Neurosci 2009;29:14408-14.

[73] Kaplan MR, Cho MH, Ullian EM, Isom LL, Levinson SR, Barres BA. Differential control of clustering of the sodium channels $\mathrm{Na}(\mathrm{v}) 1.2$ and $\mathrm{Na}(\mathrm{v}) 1.6$ at developing CNS nodes of Ranvier. Neuron 2001;30:105-19.

[74] Grubb MS, Burrone J. Activity-dependent relocation of the axon initial segment fine-tunes neuronal excitability. Nature 2010;465:1070-4.

[75] Kuba H, Oichi Y, Ohmori H. Presynaptic activity regulates $\mathrm{Na}(+)$ channel distribution at the axon initial segment. Nature 2010;465:1075-8.

[76] Schafer DP, Jha S, Liu F, Akella T, McCullough LD, Rasband MN. Disruption of the axon initial segment cytoskeleton is a new mechanism for neuronal injury. J Neurosci 2009;29:13242-54.

[77] Mantegazza M, Curia G, Biagini G, Ragsdale DS, Avoli M. Voltage-gated sodium channels as therapeutic targets in epilepsy and other neurological disorders. Lancet Neurol 2010;9:413-24.

[78] Meisler MH, O’Brien JE, Sharkey LM. Sodium channel gene family: epilepsy mutations, gene interactions and modifier effects. J Physiol 2010;588:1841-8.

[79] Smith KJ. Sodium channels and multiple sclerosis: roles in symptom production, damage and therapy. Brain Pathol 2007;17:230-42.

[80] Catterall WA, Kalume F, Oakley JC. NaV1.1 channels and epilepsy. J Physio 2010;588:1849-59.

[81] Waxman SG. Axonal conduction and injury in multiple sclerosis: the role of sodium channels. Nat Rev Neurosci 2006;7:932-41. 
[82] Craner MJ, Newcombe J, Black JA, Hartle C, Cuzner ML, Waxman SG. Molecular changes in neurons in multiple sclerosis: altered axonal expression of Nav1.2 and Nav1.6 sodium channels and $\mathrm{Na}+\mathrm{Ca}^{2+}$ exchanger. Proc Natl Acad Sci USA 2004;101:8168-73.
[83] Black JA, Dib-Hajj S, Baker D, Newcombe J, Cuzner ML, Waxman SG. Sensory neuron-specific sodium channel SNS is abnormally expressed in the brains of mice with experimental allergic encephalomyelitis and humans with multiple sclerosis. Proc Natl Acad Sci USA 2000;97:11598-602.

Please cite this article in press as: Leterrier C, et al. Determinants of voltage-gated sodium channel clustering in neurons. Semin Cell Dev Biol (2010), doi:10.1016/j.semcdb.2010.09.014 